\title{
Comparison of preoperative scores predicting outcome in elderly undergoing lung malignancies resection
}

\author{
Rita Vaz Souza, Massimiliano Bassi, Sara Mantovani, Camilla Poggi, Daniele Diso, Jacopo Vannucci, \\ Andreina Pagini, Davide Amore, Federico Venuta, Marco Anile \\ Department of Thoracic Surgery, University of Rome Sapienza, Rome, Italy \\ Contributions: (I) Conception and design: R Vaz Souza, M Bassi, M Anile; (II) Administrative support: None; (III) Provision of study materials or \\ patients: S Mantovani, C Poggi, D Diso, J Vannucci; (IV) Collection and assembly of data: A Pagini, D Amore; (V) Data analysis and interpretation: \\ M Anile, F Venuta; (VI) Manuscript writing: All authors; (VII) Final approval of manuscript: All authors. \\ Correspondence to: Camilla Poggi, MD, PhD. Department of Thoracic Surgery, University of Rome Sapienza, Policlinico Umberto I, Viale del \\ Policlinico 155, 00161 Rome, Italy. Email: camilla.poggi@uniroma1.it.
}

Background: Increased age of cancer patients is not an absolute contraindication to pulmonary resection. Different scores have been developed to determine the risk of morbidity and mortality. We have compared four scores in a series of elderly patients with primary or metastatic lung neoplasms who underwent pulmonary resection.

Methods: Data from 150 patients with an age equal or more than 75 years were reviewed. Mean age was 78.3 (range, 75-86) years. Based on medical history and preoperative tests 4 predicting scores were calculated. Statistical analysis was performed to identify which score correlates better with postoperative morbidity and mortality.

Results: Mortality at 30 days was observed in 3 patients (2\%). Postoperative morbidity was observed in 38 patients $(25.3 \%)$. Univariate analysis showed that risk factors significantly predicting the onset of postoperative complications were type of resection $(\mathrm{P}=0.02)$, American Society of Anesthesiology (ASA) score $(\mathrm{P}<0.001)$ and Glasgow Prognostic Score $(\mathrm{GPS})(\mathrm{P}=0.02)$. At multivariate analysis smoking and type of resection were significant prognostic factors for both overall and pulmonary morbidity; the ASA score and GPS showed an impact only on overall morbidity. The Cox regression showed significant results for GPS greater than zero and cancer-related death. Age above 80 years was not a negative prognostic factor. A significant difference in terms of 1-year survival was noted in ASA I-II vs. ASA III-IV (90\% vs. 78\%; $\mathrm{P}=0.022)$ and GPS 0 vs. GPS 1 or 2 (90\% vs. $77 \% ; \mathrm{P}=0.02)$.

Conclusions: Prognostic scores are useful to predict postoperative morbidity and mortality and GPS seems to correlate better with them.

Keywords: Scoring systems; elderly patients; frail patients; pulmonary resection

Submitted Apr 09, 2020. Accepted for publication Oct 25, 2020.

doi: $10.21037 /$ jtd-20-1622

View this article at: http://dx.doi.org/10.21037/jtd-20-1622

\section{Introduction}

Although old age is not actually an absolute contraindication for lung resection, aging is associated to a higher incidence of comorbidities potentially influencing the postoperative course (1). In terms of fitness for surgery, the main areas of concern are considered age, pulmonary function, cardiovascular fitness, nutrition, performance status and the presence and severity of comorbidities (2-4). Preoperative pulmonary and cardiovascular assessment is routinely performed to evaluate the surgical risk of candidates; furthermore the European Respiratory Society (ERS) and European Society of Thoracic Surgery (ESTS) guidelines recommend that, even though the use of scoring systems 
is not routinely required, these tools should be considered useful instruments for risk stratification, in particular in frail patients (5). Several preoperative scoring systems have been developed to assess frailty of elderly patients and to predict the incidence of complications and mortality (3,6-8). Each score focuses on different parameters: briefly, the American Society of Anesthesiology (ASA) physical status classification has been designed to categorize the physical status and its classes coincide with preoperative comorbidity in particular in elderly patients; the Karnofsky Performance Status (KPS) is a method through an 11-point scale (from $0 \%$ to $100 \%$ ) to assess the functional status in cancer patients before and after the treatment; the Glasgow Prognostic Score (GPS) bases on evaluation of systemic inflammatory response [calculated from the values of C-reactive protein (CRP) and serum albumin] (3) that is a major factor underlying cancerpatients decline; finally, the Charlson Comorbidity Index (CCI) considers the presence of comorbidities assigning a different weighting score and the index is the sum of all scores. We hereby report a comparison between these four commonly used scores to predict morbidity and 1-year mortality in patients older than 75 years undergoing lung resection for neoplastic diseases. We present the following article in accordance with the STROBE reporting checklist (available at http://dx.doi.org/10.21037/jtd-20-1622).

\section{Methods}

The data used in this retrospective study were collected from the medical records of 150 patients who underwent lung resection surgery between January 2013 and December 2018 at Thoracic Surgery division of Umberto I hospital. All patients have signed an informed consent to review their data. The inclusion criteria were age greater than or equal to 75 and pulmonary resection due to malignant disease, both primary lung cancer and metastatic disease. The patients were contacted by phone for the followup. The study was conducted in accordance with the Declaration of Helsinki (as revised in 2013) and approved by the institutional ethics commette of our Department (prot. 01/2019). The average age of the patients was 78.3 (range, 75-86) years with $28.7 \%$ (43 patients) of the cohort aged 80 or above. The male to female ratio was 2.2:1 (103 men and 47 women). One hundred twenty-nine patients $(86 \%)$ had a primary lung cancer, while the remaining 21 (14\%) patients were found to have lung metastases from a primary cancer elsewhere. The primary tumors in the patients with metastatic disease were: colorectal cancer
(6 patients), urothelial cancer (4 patients), breast cancer (3 patients), melanoma (2 patients), renal clear cell carcinoma (2 patients), renal leiomyosarcoma (1 patient), prostatic adenocarcinoma (1 patient), ovarian adenocarcinoma (1 patient) and thymoma (1 patient). As for the histology of the primary lung tumors, 83 (55.3\%) were adenocarcinoma, $28(21.7 \%)$ were squamous cell carcinoma and 18 (14\%) were of other histological origin, including large-cell neuroendocrine carcinoma, typical and atypical carcinoid and mixoid sarcoma. According to the $8^{\text {th }}$ edition of the TNM staging system, 84 patients $(65.1 \%)$ were diagnosed with stage I disease, 23 (17.8\%) had stage II tumors, 19 $(14.7 \%)$ were at stage III and $3(2.3 \%)$ stage IV. Since the $8^{\text {th }}$ edition only came into clinical practice in 2017, the tumor stage of all the patients operated before then was updated based on the original histological report. Of all the patients included in the study, 14 (9.3\%) underwent surgery through a VATS approach and 136 (90.7\%) underwent thoracotomy; in 68 patients $(45.3)$ we have performed a lobectomy, 64 patients $(42.7 \%)$ had a wedge resection, $5(3.3 \%)$ had lobectomy together with wedge resection, 4 (2.7\%) had bilobectomy and $9(6 \%)$ underwent pneumonectomy. Fortythree patients with primary lung cancer underwent wedge resection and the principal reason was the dimensions of the tumor that was completely removable by a sublobar resection; in only a minority of patients the choice to perform a limited resection was motivated by a poor respiratory function. Five patients with a clinical T4 (nodule satellite in other lobe) underwent lobectomy plus wedge resection; however, in only two cases the nodule satellite was confirmed as neoplastic. Only 35 (23.3\%) patients did not have a history of smoking habit with the majority (76 patients, $50.7 \%$ ) being former smokers; 39 patients (26\%) were active smokers at the time of the procedure and 14 of these patients reported an association between cigarette smoking and alcohol consumption. The overwhelming majority of patients in this study (143, $95.3 \%)$ had at least one other comorbid condition and one third of the study population reported the coexistence of at least 3 comorbidities. The medical history and the preoperative testing results were then used to calculate the four different scoring systems (Table 1). In particular, the values of CRP and serum albumin used to calculate GPS were those reported by McMillan et al. (3).

\section{Statistical analysis}

Continuous variables were expressed as mean \pm standard 
Table 1 Patients distribution on different scoring systems

\begin{tabular}{|c|c|c|}
\hline SCORE & PTS & $\%$ \\
\hline \multicolumn{3}{|l|}{ ASA } \\
\hline I & 6 & 4 \\
\hline II & 92 & 61.3 \\
\hline III & 50 & 33.3 \\
\hline IV & 2 & 1.3 \\
\hline \multicolumn{3}{|l|}{ KPS } \\
\hline$\geq 80$ & 136 & 90.7 \\
\hline$<80$ & 14 & 9.3 \\
\hline \multicolumn{3}{|l|}{ GPS } \\
\hline 0 & 105 & 70 \\
\hline 1 & 42 & 28 \\
\hline 2 & 3 & 2 \\
\hline \multicolumn{3}{|l|}{$\mathrm{CCl}$} \\
\hline $1-2$ & 51 & 34 \\
\hline $3-4$ & 56 & 37.3 \\
\hline$\geq 5$ & 43 & 28.7 \\
\hline
\end{tabular}

ASA, American Society of Anesthesiology; KPS, Karnofsky Performance Status; GPS, Glasgow Prognostic Score; CCI, Charlson Comorbidity Index.

deviation and compared by Student's test. Contingency tables with Pearson $\chi^{2}$ test and Fisher's exact test were used to compare categorical variables. Overall and diseasefree survivals were calculated with Kaplan-Meier curves. Univariate and multivariate analysis were performed in order to identify independent risk factors for survival, overall morbidity and pulmonary complications. A P value less than 0.05 was considered statistically significant. All statistical analyses were performed using SPSS 17.00 software package (Chicago, IL, USA).

\section{Results}

Postoperative morbidity was observed in 38 patients (25.3\%): prolonged air leaks (32\%), acute pulmonary edema $(5.2 \%)$, atelectasis $(21 \%)$, pneumonia $(13.1 \%)$ and respiratory failure $(2.7 \%)$ were considered as pulmonary complications. The non-respiratory postoperative complications included atrial fibrillation, anemia requiring blood transfusion, subcutaneous emphysema, myocardial infarction, chylothorax, mental status changes and infection of the surgical incision wound; atrial fibrillation was the most frequent non-respiratory complication (26.3\%). The mean hospital stay postoperatively was of $7.1 \pm 4$. days; it was longer in patients with postoperative complications ( $10.6 \pm 6.3$ vs. $5.9 \pm 1.8$ days; $\mathrm{P}=0.003)$. Mortality at 30 days was observed in 3 patients ( $2 \%$ ) but only 1 occurred during postoperative hospitalization due to a sudden cardiac arrest. Upon analysis of the contingency tables and their respective chi-square tests, it was observed that the risk factors significantly predicting the onset of postoperative complications were type of resection $(\mathrm{P}=0.02)$, ASA score $(\mathrm{P}<0.001)$ and GPS $(\mathrm{P}=0.02)$. The multivariate analysis for overall morbidity and pulmonary complications is reported in Table 2. Smoking and type of resection (lobectomy or greater) were significant prognostic factors for both overall and pulmonary morbidity; the ASA score and GPS showed an impact only on overall morbidity; CCI had a borderline value only on the onset of pulmonary complications. Age above 80 years was not a negative prognostic factor. We observed a significant difference in terms of 1-year survival in ASA I-II vs. ASA III-IV (90\% vs. 78\%; $\mathrm{P}=0.022)$ and GPS 0 vs. GPS 1 or 2 (90\% vs. 77\%; $\mathrm{P}=0.02$ ) (Figures 1,2). The results of the multivariate cox regression analysis for survival are reported in Table 3. We observed that the variables that significantly influence long-term survival are cancer-related death and GPS. The type of resection performed (wedge resection versus greater resections) showed a borderline significance. The average length of follow-up was 24.5 months (range, 2-67 months). Nine patients $(6 \%)$ were excluded from the dataset used in the statistical analysis due to the inability to contact them or a family member (considered lost at follow-up).

\section{Discussion}

Preoperative scoring systems are widely used to predict postoperative morbidity and mortality (2), particularly in frail patients like the elderly population; however, a comparison between these scores has been not reported yet. In our study, ASA score and GPS seem to show a strong impact to predict the onset of postoperative complications. While the significance of the GPS in predicting morbidity, also with modified values of CRP and serum albumin (9), is in accordance with previous studies (3), the impact of ASA score is still debatable (5) since it is less effective compared to other scoring systems; it has, however, been shown to be a useful measure of comorbidity (6), which in itself is a well-reported predictor of postoperative complications, 
Table 2 Multivariate logistic regression for overall morbidity and pulmonary complications

\begin{tabular}{|c|c|c|c|c|c|c|}
\hline Variables & \multicolumn{3}{|c|}{ Overall morbidity } & \multicolumn{3}{|c|}{ Pulmonary complications } \\
\hline Gender & 2.304 & $0.781-6.801$ & 0.1 & 2.073 & $0.537-8.004$ & 0.2 \\
\hline Age $>80$ & 1.411 & $0.532-3.741$ & 0.4 & 2.674 & $0.813-8.798$ & 0.1 \\
\hline Smoking & 4.686 & $1.225-17.923$ & $0.02^{*}$ & 20.90 & $1.818-240.44$ & $0.01^{*}$ \\
\hline$S+A$ & 0.421 & $0.062-2.862$ & 0.3 & 0.987 & $0.142-3.876$ & 0.4 \\
\hline FEV1 & 2.008 & $0.736-5.474$ & 0.1 & 1.332 & $0.378-4.690$ & 0.6 \\
\hline Surgery & 6.600 & $2.297-18.968$ & $<0.0001^{*}$ & 6.680 & $1.774-25.154$ & $0.005^{*}$ \\
\hline ASA & 5.478 & $2.003-14.985$ & $0.001^{*}$ & 1.859 & $0.541-6.388$ & 0.3 \\
\hline $\mathrm{CCl}$ & 1.170 & $0.606-5.173$ & 0.2 & 3.614 & $0.924-14.132$ & $0.055^{\S}$ \\
\hline
\end{tabular}

*, statistical significance; ${ }^{\S}$, borderline significance. ASA, American Society of Anesthesiology; KPS, Karnofsky Performance Status; GPS, Glasgow Prognostic Score; CCl, Charlson Comorbidity Index; S+A, smoking + alcohol; FEV , forced expiratory volume in 1 Second.

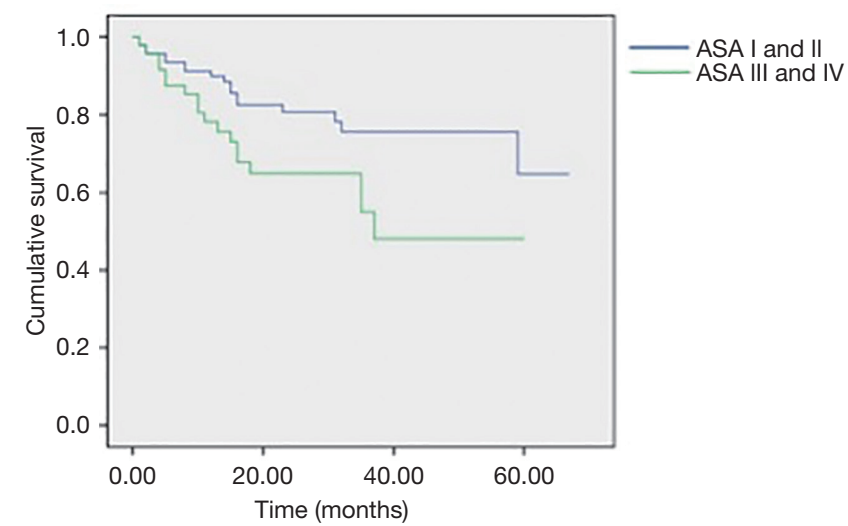

Figure 1 Kaplan-Meier curve showing survival related to American Society of Anesthesiology (ASA) score ( $\mathrm{P}=0.022)$.

although this is more often represented as the CCI, rather than ASA score. Studies by Birim et al. (10) and Rueth et al. (11) concluded that the CCI was the only one of the analyzed variables that significantly predicted postoperative morbidity. In our study, however, a CCI greater than or equal to 5 was not shown to be a risk factor for complications. When evaluating which factors predict mortality, the univariate analyses showed that the only significant variables were ASA score greater than 2 and GPS greater than zero, with $\mathrm{CCI} \geq 5$ being on the borderline of significance. However,

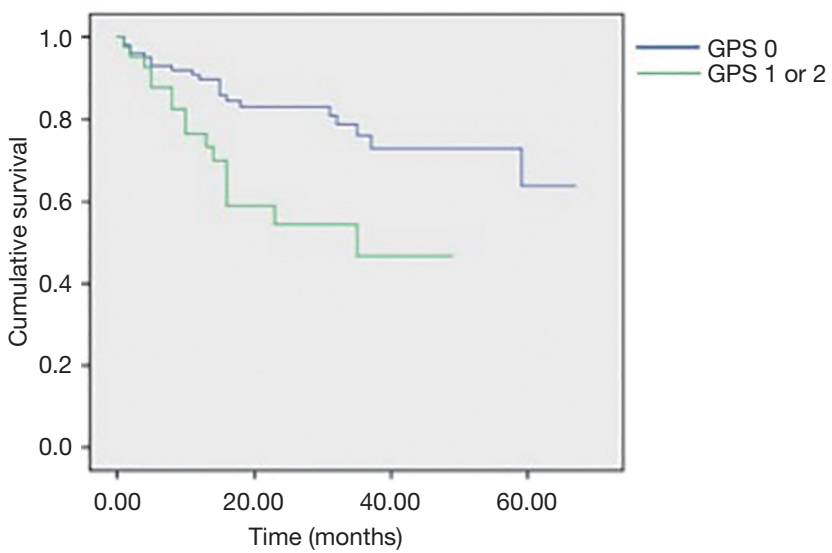

Figure 2 Kaplan-Meier curve showing survival related to Glasgow Prognostic Score (GPS) (P=0.02).

the multivariate Cox regression only yielded significant results for GPS greater than zero and cancer-related death; lobectomy and greater resections were at the borderline of significance. In published literature, we can find results contradictory to these. Different studies have reported statistical significance of stage, smoking history, $\mathrm{FEV}_{1}$ value, ASA score, CCI and postoperative morbidity in predicting overall survival $(6,8,10,12)$, but none of these studies evaluated the significance of the GPS. The relevance of the GPS as a prognostic indicator in cancer patients is widely 
Table 3 Multivariate Cox regression for survival

\begin{tabular}{lccc}
\hline Variable & Hazard Ratio & $95 \% \mathrm{Cl}$ & P value \\
\hline Gender & 0.84 & $0.32-2.22$ & 0.72 \\
Approach & 0.78 & $0.10-6.07$ & 0.81 \\
KPS & 1.18 & $0.34-4.02$ & 0.80 \\
FEV 1 & 1.10 & $0.42-2.87$ & 0.85 \\
Morbidity & 1.34 & $0.58-3.10$ & 0.49 \\
Smoking history & 0.52 & $0.20-1.32$ & 0.17 \\
Cancer-related & 21.88 & $8.70-54.99$ & $<0.001$ \\
death & & & \\
Tumor stage & 0.78 & $0.32-1.88$ & 0.58 \\
ASA Score & 1.35 & $0.62-2.91$ & 0.45 \\
Type of resection & 0.41 & $0.17-1.01$ & 0.05 \\
GPS & 3.27 & $1.49-7.18$ & 0.003 \\
CCI & 0.60 & $0.25-1.47$ & 0.27 \\
\hline
\end{tabular}

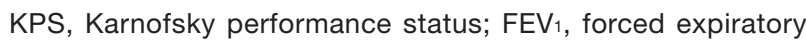
volume in 1 second; ASA, American Society of Anesthesiologists; GPS, Glasgow prognostic score; $\mathrm{CCl}$, Charlson comorbidity index.

established and it is considered a useful predictor of both morbidity and mortality (3). Finally, patients with ASA score III-IV and GPS 1-2 show a higher 1-year mortality risk.

Interestingly, the tumor stage did not appear to be a significant predictor of mortality in either type of analysis. However, when stratified against the ASA score, it was observed that patients with an ASA score greater than 2 and with advanced tumor stage (III or IV) have a significantly lower long-term survival rate than those with early-stage tumors. Therefore, we can conclude that tumor stage is in fact predictive of mortality in patients with poor physical status. It has been suggested that sublobar resections are predictive of reduced survival due to the possibility of incomplete removal of the tumor and consequent recurrence of the disease (13). However, the effect of the type of resection on survival seems to be age-related. Several studies concluded that there is no difference in the survival of elderly patients who undergo a limited resection compared to those who undergo lobectomy $(5,6,10,14,15)$. Pneumonectomy, on the other hand, is associated with significantly higher morbidity and mortality among the elderly $(5,12,14)$. To further support this correlation between extent of resection, mortality and age, the study by Mery et al. (14) reported that below 71 years of age, the difference in survival among patients who undergo wedge resection versus those who undergo lobectomy does become significant. We can therefore conclude that the results of our study are in accordance with the published literature in that, for patients of age 75 years or above, undergoing a wedge resection as opposed to a more extensive resection does not have a statistically significant impact on long-term survival. However, an analysis of the effect of pneumonectomy on survival is lacking and was not performed since only 9 patients $(6 \%)$ of the cohort underwent this procedure.

Surgical resection of either primary lung cancer or metastatic disease in elderly patients confers good longterm survival. As such, it should be offered as a treatment option to patients deemed fit for surgery, regardless of age. Based on the results of this study, the best predictor for both morbidity and mortality is the GPS and its use could be of help in determining which patients require closer monitoring in the postoperative period. Another factor that should be given particular significance is the ASA status, especially regarding its potential for predicting complications. However, because there are discrepancies between the results reported in this study and those from larger multi-center studies, the importance of factors such as tumor stage and comorbidities cannot be ignored when evaluating the prognosis of the patients.

\section{Acknowledgments}

Funding: None.

\section{Footnote}

Reporting Checklist: The authors have completed the STROBE reporting checklist. Available at http://dx.doi. org/10.21037/jtd-20-1622

Data Sharing Statement: Available at http://dx.doi. org/10.21037/jtd-20-1622

Conflicts of Interest: All authors have completed the ICMJE uniform disclosure form (available at http://dx.doi. org/10.21037/jtd-20-1622). CP serves as an unpaid editorial board member of Fournal of Thoracic Disease from May 2017 to Jul 2021. FV serves as an unpaid editorial board member of Fournal of Thoracic Disease from Jun 2014 to Jul 2021. The other authors have no conflicts of interest to declare.

Ethical Statement: The authors are accountable for all 
aspects of the work in ensuring that questions related to the accuracy or integrity of any part of the work are appropriately investigated and resolved. The study was conducted in accordance with the Declaration of Helsinki (as revised in 2013) and approved by the institutional ethics committee of our Department (prot. 01/2019). All patients have signed an informed consent to review their data.

Open Access Statement: This is an Open Access article distributed in accordance with the Creative Commons Attribution-NonCommercial-NoDerivs 4.0 International License (CC BY-NC-ND 4.0), which permits the noncommercial replication and distribution of the article with the strict proviso that no changes or edits are made and the original work is properly cited (including links to both the formal publication through the relevant DOI and the license). See: https://creativecommons.org/licenses/by-nc-nd/4.0/.

\section{References}

1. Venuta F, Diso D, Onorati I, et al. Lung cancer in elderly patients. J Thorac Dis 2016;8:S908-14.

2. Brunelli A, Charloux A, Bolliger CT, et al. ERS/ESTS clinical guidelines on fitness for radical therapy in lung cancer patients (surgery and chemo-radiotherapy). Eur Respir J 2009;34:17-41.

3. McMillan DC. The systemic inflammation-based Glasgow Prognostic Score: a decade of experience in patients with cancer. Cancer Treat Rev 2013;39:534-40.

4. Saji H, Ueno T, Nakamura H, et al. A proposal comprehensive risk scoring system for predicting postoperative complications in octagenarian patients with medically operable lung cancer: JACS1303. Eur J Cardiothorac Surg 2018;53:835-41.

5. Gonzalez Aragoneses F, Moreno-Mata N, Simon-Adiego $\mathrm{C}$, et al. Lung cancer surgery in the elderly. Crit Rev Oncol Hematol 2009;71:266-71.

6. Brock MV, Kim MP, Hooker CH, et al. Pulmonary resection in octogenarians with stage I non-small cell lung cancer: A 22-year experience. Ann Thorac Surg

Cite this article as: Vaz Souza R, Bassi M, Mantovani S, Poggi C, Diso D, Vannucci J, Pagini A, Amore D, Venuta F, Anile $M$. Comparison of preoperative scores predicting outcome in elderly undergoing lung malignancies resection. J Thorac Dis 2020;12(12):7083-7088. doi: 10.21037/jtd-20-1622
2004;77:271-7.

7. Péus D, Newcomb N, Hofer S. Appraisal of the Karnofsky Performance Status and proposal of a simple algorithmic system for its evaluation. BMC Medical Informatics and Decision Making 2013;13:72-8.

8. Yang CC, Fong Y, Lin LC, et al. The age-adjusted Charlson Comorbidity Index is a better predictor of survival in operated lung cancer patients than the Charlson and Elixhauser Comorbidity Indices. European Journal of Cardio-Thoracic Surgery 2018;53:235-40.

9. Kawashima M, Murakawa T, Shinozaki T, et al. Significance of the Glasgow Prognostic Score as a progenostic indicator for lung cancer surgery. Interact CardioVasc Thorac Surg 2015;21:637-43.

10. Birim O, Zuydendorp M, Maat APWM, et al. Lung resection for non-small-cell lung cancer in patients older than 70: mortality, morbidity, and late survival compared with the general population. Ann Thorac Surg 2003;76:1796-801.

11. Rueth NM, Parsons HM, Habermann EB, et al. Surgical treatment of lung cancer: predicting postoperative morbidity in the elderly population. J Thorac Cardiovasc Surg 2012;143:1314-23.

12. Okamoto J, Kubokura H, Usuda J. Factors determining the choice of surgical procedure in elderly patients with non-small cell lung cancer. Ann Thorac Cardiovasc Surg 2016;22:131-8.

13. Khullar OV, Liu Y, Gillespie T et al. Survival after sublobar resection versus lobectomy for clinical stage IA lung cancer: an analysis from the National Cancer Data Base. J Thorac Oncol 2015;10:1625-33.

14. Mery CM, Pappas AN, Bueno R, et al. Similar long-term survival of elderly patients with non-small cell lung cancer treated with lobectomy or wedge resection within the Surveillance, Epidemiology, and End Results Database. Chest 2005;128:237-45.

15. Jaklitsch MT, Pappas-Estocin A, Bueno R. Thoracoscopic surgery in elderly lung cancer patients. Crit Rev Oncol Hematol 2004;49:165-71. 